\title{
Semiotic Training for Brain-Computer Interfaces
}

\author{
Mariya Timofeeva \\ Sobolev Institute of Mathematics SB RAS, \\ 4 Acad. Koptyug Avenue, \\ Novosibirsk State University, \\ 2 Pirogova Str. 630090 Novosibirsk, \\ Russian Federation \\ Email: timof@math.nsc.ru
}

\begin{abstract}
With time education becomes more personified. New categories of learners join in educational processes and new areas of education appear. Brain-computer interfaces have good perspective to contribute to these tendencies. This technology may allow disabled people to participate in social life, including education, and may let healthy people to develop the skill of controlling brain waves. Training the skill is the object of investigation and researchers recommend taking into account human factors: general principles of learning, motivations, personal patterns and abilities (among them, spatial). Education may be a source of highly motivated training tasks. The paper treats brain-computer interface as a type of communication and argues for semiotic training that is a variant of training spatial abilities. Semiotic training have proved effectiveness in other areas of education. Theoretical background and preliminary empirical comments of the approach are considered.
\end{abstract}

\section{INTRODUCTION}

$\mathrm{T}$ HE aim of the paper consists in attracting attention to educational perspectives connected with future development of brain-computer interfaces (BCI) and proposing theoretical rationale for semiotic training as a possible way of developing the skill of controlling brain waves. BCI is considered as a sort of communication.

\section{Structure of the Paper}

Consequent reasoning characterizes the trends in education and introduces the aspects of BCI that are important for the issue under consideration (Section II). Section III presents general information about BCI. Section IV proposes semiotic view on BCI, argues for methodology of field linguistics as the perspective way of learning BCI skill; discusses basic lines of semiotic training and its benefits. Section $\mathrm{V}$ proposes the illustrative model by considering semiotic regularities of human pictorial imagination appearing in comics, the other products of human intentional imagination. The model analysis provides data that may be useful for strategies of semiotic training. Section VI resumes the considered issue.

\section{TRENDS IN EDUCATION}

Three trends of contemporary education are especially important for the current consideration: individualization of ed-

I am very grateful to Sobolev Institute of Mathematics and Novosibirsk State University for supporting my research ucational trajectories, widening the scope of educational activity, and involving new categories of learners.

The scope of educational activity have substantially widened when its virtual forms appeared and allowed new categories of learners to participate in e-learning. Besides, education tends to become more individually fitted and polyvariant. Strategies of education come closer to self-education. Subjectively estimated, elaborated and regulated ways of developing knowledge and experience gain their significance for the subjects of education. Not only general solutions concerning perspectives of education in whole are significant, specific educational technologies designed for concrete category of learners are also valuable.

Developing BCI seems to have a strong potential of contributing to these trends. Communication in whole is basic for education, thus training $\mathrm{BCI}$ as the specific means of communicating with external world for mastering internal skills can involve certain categories of people (disabled or healthy) into educational processes [1]-[4]. Besides, according to [5], reproducing physical actions (with the help of movement interactive devices) trains memory and motor abilities. This result supports the hypothesis that implementing physical actions by the force of imagination may give the same benefits. The proposed semiotic training procedure may give double benefit by affecting both semiotic and brain control skills. The former is valuable for dealing with semiotic systems (for instance, natural languages), the latter - for mastering BCI.

\section{BRAIN-COMPUTER INTERFACES}

\section{General Characteristics}

$\mathrm{BCI}$ allows a person to perform physical actions (on the screen or really) by the force of intended imagination, or more precisely, by the force of another side of imagination brain waves - that are fixed and translated into characteristics of the physical action. The intentions often are not fully arbitrary, and should follow a task that is given to a BCI user. The tasks may differ in complexity and content. Some $\mathrm{BCI}$ designs presuppose a free training session. During this session, a BCI user chooses imagery tasks by his / her own decision [6].

Among the current discussions about BCI, two items are especially crucial for prospective education. 
First, training procedures for mastering this sort of interaction, particularly mental tasks and feedbacks. Currently used training protocols are discussed, for instance, in [6]. Feedback provides perceivable data for accessing effectiveness of brain control. There are different variants of feedback, for instance, neurofeedback [7], the motor imagery training system proposed in [8]. The present paper discusses only the variant of training BCI skill and does not concern the details of designing feedbacks.

Second, perspectives of BCI that go beyond solely medical purposes and cover other sorts of activities intended for disabled or healthy people [1]-[2].

BCI is substantially interdisciplinary undertaking and, as the authors of [9] notice, cooperation across disciplines has good potential to improve situation. The present paper proposes linguistic view on the situation.

This view rests on considering BCI as a sort of communication subject to semiotic interpretation. The stance suggests the idea of semiotic training based on the methodology of field linguistics. In case of BCI, the semiotic training will develop spatial abilities. This is important because according to the hypothesis [10]-[11] spatial abilities may efficiently contribute to the development of BCI skill.

\section{Training Procedures}

Efficiency of BCI may depend on ability of a human to control brain activity, and this skill is not inherent by nature, many people (between 15 and $30 \%$ [10]) cannot use it at all. It is crucial for certain types of BCI, particularly for spontaneous BCI [6]. Lack of the capacity to control brain waves is called "BCI illiteracy" or "BCI deficiency" [10].

Thus, thinking over strategies of preliminary training and perfecting the capacity is often marked as actual. Two approaches are noteworthy in the context of the present paper. The first [6] recommends to generalize the key features of efficient training in different areas of knowledge and to infer relatively skill-independent recommendations. The second [10] suggests personifying the process by using individually designed strategies of mastering the skill. Since we consider $\mathrm{BCI}$ as a sort of communication, the former approach gives reason for generalizing linguistic experience of learning unknown languages by reconstructing the semiotic structure of a language on the base of the speech data. According to the latter approach individual semiotic training (as a skill of reconstructing semiotic structures from communicative data) seems to be promising.

\section{SEMIOTIC VIEW ON BRAIN-COMPUTER INTERFACE}

\section{Brain-Computer Interface as a Semiotic System}

BCI is interpretable as a sort of translation that transforms information along the following line: intention - (visual or kinesthetic) image of a desired physical action - brain waves - specification of the action (recognized by special equipment and appropriate algorithms) - physical action. A user of BCI cannot monitor directly brain waves; nevertheless, (due to perceivable feedback) he/she may regulate it indirectly, through modifying intentions and therefore images. Thus for a user of BCI the aforementioned line of translation is shorter: intention - image - physical action. The shortened line admits semiotic representation.

The notion of sign varies in different semiotic theories. "Dyadic" [12] and "triadic" [13] models of sign are the most common. The visual form of the latter is "semiotic triangle". There is no full agreement about the terms marking the interrelated angles of the triangle. We will use the following terms: sign (signifier, a linguistic form) - meaning (mental entity, concept) - reference (physical or abstract entity indicated by the sign in a real act of communication). A language user can directly control the usage of a sign and voluntary modify it if necessary.

In BCI, image plays the role of a signifier. A BCI user can intentionally modify it. Resulting physical action plays the role of the referent Meaning is individual intrinsic skill of building appropriate images, i.e. images that effectively initiate desired actions.

An image may be a simple sign or a compound sign. The components of a compound sign correlate with the features of a referent. Compound signs appear on rather developed level of communicative skill.

In linguistics, natural language signs are usually considered as linear, one-dimensional. In BCI, signs are non-linear. BCI as communication is closer to sign languages, (used by deaf people): sign languages localize in space, not in line.

\section{Extractability of Semiotic System}

Semiotic systems can vary in their substance broadly. They are not limited in modalities of realization, in number of dimensions, or nature of elementary and complex signs. At the same time, there are general rules governing their internal structure and functioning, and these two aspects are closely connected: a structure becomes apparent during functioning and thus is extractable from the instances of functioning. Validity of the result depends on quantity and quality of communicative data.

Sign languages give the appropriate illustration. These languages are spatial: a "speaker" creates signs by different sorts of gestures, including body movements and mimics. Thus, competence in a sign language requires sufficiently developed spatial abilities.

The history of sign languages is rather instructive. Many years had passed before linguists recognized semiotic nature of sign languages and began to consider them not as a mere pantomime. This happened due to the pioneer work of William Stokoe [14]; in $1960^{\text {th }}$ sign languages became the objects of contemporary linguistics. Possibility of the crucial turn was stipulated by semiotic analysis fulfilled by Stokoe: he extracted constituents of semiotic system ("cheremes") from continuum of raw communicative data. This process is similar to deciphering a language. Corresponding linguistic methodology is typical for field linguistics.

Viewing the gesture space as a medium obeying semiotic regularities permits to segment gestures into elementary features of signs and to imitate (to a certain extent) communication based on a sign language automatically in the systems of machine translation [15].

Theoretically, an intended image in BCI can also be semiotically complex and consist of several more simple parts 
of signs. Presumably, these constituents of the intended image correspond to certain constituents of the desired resulting movement; and a set of such constituents is individual and specific for each person.

Special area of linguistics develops methodology for extracting semiotic systems from raw communicative data. This is "field linguistics" [16].

\section{Field Linguistics}

Field linguists are learning and studying the languages (usually exotic) that are unwritten, familiar only to their native speakers who do not know any other language besides their own. Moreover, the circumstances of life and phonetic features of the target language are also exotic thus limiting linguists in using analogies.

Field linguist works in specific investigational situation when the researcher has no linguistic competence in the target language, and the native speaker constitutes the only source of information about the language [16]. Similarly, a BCI user fulfills the role of a "linguist", which is analyzing perceptive data in order to extract the underlying semiotic patterns.

Both types of communicators, intentionally and experimentally, by trial and error, are trying to detect (rationally or only perceptually) the proper abstract knowledge. Difference is in the nature of this knowledge: a BCI speaker develops visual or kinesthetic skill, a linguist articulatory skill that also can be auditory or kinesthetic. In both cases kinesthetic skill is said to be more basic ([6], [16]).

At the very beginning of a scientific research (within so called "zero cycle"), a field linguist knows nothing about semiotic structure of the target language, he / she should discover the signs and meanings of the language on the base of analyzing language use.

Undoubtedly, BCI speaker usually is not a linguist; these two types of people have different interests and goals. Nevertheless, the linguistic character of BCI training is substantially similar to that of a zero cycle in field linguistics. Both are akin to deciphering.

\section{Semiotic Training}

If a BCI user wants to develop the skill of controlling brain waves, he / she tries to discover why the image of desirable movement appeared to be insufficiently strong. Actually, the process means that the BCI user tries to change hypothesis about the proper constituents of the images (signs) and to elaborate the more effective ones. Developing BCI skill actually means the process of refining the hypothesis. In fact, a BCI user intentionally trains his / her linguistic competence in BCI communication. The field linguist fulfills the similar task.

The learning procedures for developing semiotic skill should train general subject independent schemas of semiotic analysis; at the same time, the object of the analysis is individual (individual perceptive data). In contrast, a foreign language learner usually learn the concrete semiotic system with previously stated signs.

Semiotic training involves series of iterative semiotic analysis. The latter consists in searching for combinatorial regularities in perceptive data. General regularities are common and sufficiently strict; [17] presents basic variant of the procedures used in field linguistics. The whole scope of investigation in field linguistics embraces all language levels and thus may seem not easy for a BCI user if he / she is not a linguist. Nevertheless, semiotic analysis is flexible and does not require building a multilevel structure (as for natural languages); the analysis may not go beyond one level thus becoming well understandable.

The proposed procedure of semiotic analysis is a sort of mental experiment. A user of BCI, or an "experimenter", may realize it by fulfilling the following types of operations: 1) to fix some part of an image of the desired physical action; 2) to vary the remained part / parts and simultaneously trace the results (check if the resulting physical action is appropriate); 3) to repeat (if necessary) the experiment with different variants of the division; 4) to search for interchangeable parts that may replace each other without changing the context (the sets of such parts hypothetically correspond to abstract entities).

The experimenter may repeat these operations cyclically and then summarize the results of several experiments. The obtained system of signs will be individual and specific for each person.

It is worth noting that each semiotic cycle exercises spatial abilities of the person.

Semiotic training may give double benefit by affecting both brain control skill and semiotic skill.

The latter is substantial for dealing with other semiotic systems, particularly with natural languages, and this idea has the empirical confirmation. The Traditional Linguistics Olympiads successfully use the linguistic variant of supposed semiotic tasks (in the spirit of field linguistics) for searching and training linguistically gifted children. For solving the tasks of the Olympiad, no prior knowledge of linguistics or languages is required: logical ability and the will are sufficient. Information about the Olympiads and the collections of tasks are available at http://www.ioling.org/, http://www.lingling.ru/olymps/mos_olymp/. Several decades (46 years) of fruitful practicing this type of semiotic tasks give empirical support for efficiency of training procedures based on the methods of field linguistics.

\section{V.SEMIOTIC ANALYSIS}

\section{Parameters of Images}

Semiotic analysis briefly depicted in the previous section does not provide deterministic procedure. The analysis has no less than four degrees of freedom: 1) the way of segmentation and granularity of the static perceived data, 2) the way of segmentation and granularity of the dynamic perceived data, 3) the set of simple / elementary actions, and 4) the goals of actions. For instance, a BCI user fulfilling the task of grasping an apple may imagine only a rather undetermined and undivided movement beginning from the initial location of the hand and ending at the location of the apple. In other case, the user may imagine the same process in details including, for instance, the images of tensed muscles, trajectory of hand movements, positions of fingers on the apple, rotation of the apple. The whole task may correspond to one goal or to the 
set of interconnected goals, for instance: to bring the hand nearer to an apple, to open the fingers, to touch the apple by the palm, to clasp the fingers around the apple.

Thus, semiotic analysis may be characterized by certain numeric parameters, among them: number of discrete parts detected in the visual continuum; number of modified parts; number of steps implementing the modifications; power of the set of elementary static / dynamic constituents. A person may determine these parameters of imagination by his / her own choice.

Nevertheless, semiotic analysis depend not only on individual decisions; it may reflect some general regularities: limitations, peculiarities and habits of people. Semiotic analysis made by linguists cannot give an appropriate example for learning these regularities because linguists usually have overtrained semiotic skill; a BCI user, on the contrary, may have a minimal experience in semiotic analysis. Besides, linguists as a rule do not analyze dynamic imagination. According to the survey of linguistic approaches to comics [18], the works in this area usually use comics for supporting the already existing linguistic theories elaborated for ordinary human languages. For our aims, we need to understand how the mind transfers meanings from imaginary to visual modality.

It would be useful to have some preliminary considerations about the parameters. These considerations may help to specify initial point for launching a semiotic cycle and to propose some guidelines for its developing.

It is not easy to undertake wide-ranging semiotic investigation of BCI data. Contemporary BCI is still barely used outside laboratories [10]-[11] and thus does not allow to observe diversified and complex physical movements implemented by brain force. Therefore, it is productive to make preliminary observations concerning the abovementioned substantial features of human intentional imagination on the base of other data that are more easily acceptable and do not need sophisticated or expensive equipment. We suppose to consider pictorial imagination for illustrative model analysis.

\section{Model Analysis}

The main question for consideration: How do a person split imaginary actions into parts when he / she reproduces the copies of the actions in the physical world?

The purposes of model analysis are: 1) to refine the list of parameters substantial for visualizing imaginary actions; 2) to provide data useful for operating with the parameters. Primarily we should choose a type of pictorial imagination appropriate for the purposes of model analysis.

As [19] shows, the way of performing a spatial task depends on the medium where it is performed. If one performs the task in virtual world, on the screen, the operations should be precise, discrete, well planned, governed by the goal in mind. The details of performing operations should be rather explicit than implicit. On the contrary, in the real world, one performs the same task intuitively, operations are continuous, and do not require high degree of specification. The image of a future action often appears in consciousness only vaguely. The authors of [19] suggest that spatial thinking skills required for creating representations in virtual and real worlds may be different. Actions performed due to BCI, like actions in virtual world, require higher degree of refinement.

In this respect, painting gives a similar example because for depicting an imagined scene, a painter should refine the image (initially viewed as integral and continuous) and may divide it into discernible well-defined parts. A picture alone is static, yet a sequence of pictures may depict dynamic stages of an action. This is typical for comics, thus comics give appropriate illustrative model for considering regularities in visual representations of imagined actions.

A person implementing a complex action by the force of imagination divides the action into parts. The painter of a comics also transforms a continuous action into discrete parts, i.e. determines, among other things, the static and dynamic components of the image, the elementary movements, the goals and sub-goals of actions; the abstract characteristics like speed, chronological order, importance, intensity. In other words, the painter makes decisions similar to these that are crucial for implementation of a physical action within BCI.

Comics may vary broadly in their genres. For our purposes, we need an example with a realistic plot, which depicts coherent realizable situations and processes. Logicomix [20] is the suitable variant for our model analysis.

The analysis showed that the way of splitting actions into parts depends rather on distribution of attention than on type of action. Attention may focus on the stages of an action and / or on its characteristics (particularly, abstract). For instance, intention to attract attention to quickness of an action often results in dividing the action into stages, i.e. fixing the successive phases of the action. This mode of emphasizing the speed / intensity may be used with reference to physical or to internal actions (for instance, mental).

Thus, the main recommendation for developing semiotic abilities may consist in training the skill of distributing degrees of attention between actions, their stages and characteristics. The skill presupposes elaborating the subjective patterns of simple actions (stages of actions) typical for essential behavior and training these patterns separately, as the isolated signs; stages are especially actual for intensive / high-speed actions. It may be useful to ask a person who wants to develop semiotic skill to make a drawing of the stages of a desired action. However, for disabled people this variant may be impossible. Concrete material for preliminary designing patterns and splitting actions is extractable from the typology of actions.

The analyzed material induces the ternary typology of actions that include active states, detailed actions, and undetailed actions.

Active states include: a) monotonic repeated movements, b) random movements specific for a situation under consideration, c) panoramic view of co-located motions. One active state usually corresponds to one panel.

For instance, several elementary movements on one panel may correspond to a continuous monotonous process like walking round and round a flowerbed during a course of cogitation. The panel in this case shows several positions of the person located on the garden path around the flowerbed. Monotonous repeated processes like dangling a foot give 
another example of elementary movements representable on one panel; in this case, the panel shows several positions of the foot simultaneously. Both cases give evidences for relatively small importance of elementary movements; the movements from one panel have common purpose.

The detailed action has refinements that may indicate stages, chronological order, highlighted constituent parts, speed, and degree of importance or intensity. Indicators of these characteristics include assortment of colors (the past is less colored), focus distance (may reflect degree of importance, intensity, speed); rotations (may serve as a means of highlighting the actual constituents). Little difference between successive panels may indicate intensity of an action (predominantly for mental actions). Visual indicators of changing attention may include; moving away from a depicted scene or closer to it (making an image, not frame, smaller / larger); modifying the angle of the field of vision; modifying the environment, elimination of the background (thus emphasizing the focus of attention).

In addition to the parameters listed above the annotation of the panels includes information about the hierarchy and the types of physical actions.

The whole slot of the comics breaks up to sub-slots (scenes); a sub-slot depicts a sequence of collocated actions. In Logicomix, the volume a sub-slot may reach several dozens of panels.

Realization of a complex action may include several simple actions (the stages). A simple action may include several elementary movements; a panel presents all of them simultaneously. Thus, we have the following hierarchy: slot sub-slots - complex actions - simple actions - elementary movements. The hierarchy of actions corresponds to the hierarchy of goals.

The database provides characteristics actual for semiotic training (calibration, launching and developing semiotic analysis); proposes the ways of visualizing complex actions and their characteristics (particularly, abstract) for designing $\mathrm{BCI}$ and other types of visualized human-computer interfaces.

\section{CONCLUSION}

$\mathrm{BCI}$ and the skill of controlling brain waves are prospective areas of future education. This position, expressed, for instance, in [3] and [4], is the starting point of the paper.

BCI is a sort of communication and allows semiotic analysis. The proposed semiotic training procedure bases on the linguistic experience elaborated for dealing with unknown semiotic systems. Semiotic training (in its linguistic version) has already proved its effectiveness in linguistic education.

The realized model analysis considers pictorial intentional imagination as a source of preliminary data useful for developing semiotic analysis.

The proposed variant of semiotic training is consistent with general recommendations that the specialists in BCI suggest: it takes into account human factors, individual capacities, and trains spatial abilities of a person.

Semiotic training may have different implementations; its potential seems to be promising and going beyond the area of $\mathrm{BCI}$ or training the skill of controlling brain waves.

\section{REFERENCES}

[1] Jan B.F. Van Erp, F. Lotte, M. Tangermann, "Brain-computer interfaces: beyond medical applications", Computer -IEEE Computer Society-, IEEE, 2012, $45 \quad$ (4), pp.26-34. http://doi.ieeecomputersociety.org/10.1109/MC.2012.107.

[2] C. Ring, A. Cooke, M. Kaussanu, D. Mclntyre, R. Masters, "Investigating the efficiency of neurofeedback training for expediting expertise and excellence in sport", in Psychology of sport and exercise, vol. 16, part 1, January 2015, pp. 118-127. DOI:10.1016/j.psychsport.2014.08.005.

[3] B. Sabitzer, "Neurodidactics - a new stimulus in ICT and computer science education", INTED2011 Proceedings, 2011, pp. 5881-5889.

[4] M. Ferrari and H. McBride, "Mind, Brain, and Education: The Birth of a New Science", in LEARNing Landscapes, vol. 5, No. 1, autumn 2011, pp. 85-100.

[5] J. E. Garrido, V. M. R. Penichet, M. D. Lozano and L. A. Sánchez, "Mobility and memory training through movement interaction," Computer Science and Information Systems (FedCSIS), 2012 Federated Conference on, Wroclaw, 2012, pp. 883-889.

[6] F. Lotte, F. Larrue, Ch. Mühl, "Flaws in current human training protocols for spontaneous brain-computer interfaces: lessons learned from instructional design", in Frontiers in Human Neuroscience, 2013, vol. 7, N 568 (11 p.). DOI: 10.3389/fnhum.2013.00568.

[7] C. Neuper and G. Pfurtscheller, "Neurofeedback training for BCI control", in Brain-computer interfaces, Eds. B. Graimann, G. Pfurtscheller and B. Allison, London: Springer, 2010, pp. 65-78. DOI: 10.1007/978-3-642-02091-9 4.

[8] H.-J. Hwang, K. Kwon, and C.-H. Im, "Neurofeedback-based motor imagery training for brain-computer interface (BCI)", in Journal of neuroscience methods, vol. 179, issue 1, April 2009. Pp. 150-156. DOI: 10.1016/j.jneumeth.2009.01.015.

[9] Brain-computer interfaces. Revolutionizing human-computer interaction. The Frontiers Collection. Eds. B. Graimann, G. Pfurtscheller and B. Allison, London: Springer, 2010, 393 p. DOI: 10.1007/978-3-642-02091-9.

[10] C. Jeunet, B. N'Kaoua, S. Subramanian, M. Hacher, and F. Lotte, "Predicting mental imagery-based BCI performance from personality, cognitive profile and neurophysiological patterns", PLOS ONE, vol. 10 (12): Dec. 2015. DOI: http://dx.doi.org/10.1371/journal.pone.0143962.

[11] F. Lotte, C. Jeunet, "Towards improved BCI based on human learning principles", $3^{\text {rd }}$ International Winter Conference on Brain-Computer Interfaces, High1 Resort, South Korea, Jan. 2015, < hal-01111843>. DOI: 10.1109/IWW-BCI.2015.7073024.

[12] F. de Saussure, Course in general linguistics, trans. Roy Harris, [1916] London: Duckworth, 1983.

[13] C. S. Peirce, Collected writings (8 Vols.), Ed. Charles Hartshorne, Paul Weiss and Arthur W. Burks, Cambridge, MA: Harvard University Press, 1931-58.

[14] W. C. Stokoe, "Sign Language structure: an outline of the visual communication systems of the American deaf", in J. of Deaf Studies and Deaf Education. 2005; 10(1), pp. 3-37. DOI: 10.1093/deafed/eni001.

[15] M. Huenerfauth, "A survey and critique of American Sign Language natural language generation and machine translation systems", Technical Report MS-CIS-03-32, Computer and Information Science, University of Pennsylvania, 2003, 36 p.

[16] A. Kibrik, The methodology of field investigations in linguistics (setting up the problem), Janua Linguarum. Series Minor, 142, The Hague/Paris: Mouton, 1977, 130 p.

[17] H. Gleason, An introduction to descriptive linguistics, rev. ed., New York: Holt. Rinehart \& Winston, 1961, 503 p.

[18] N. Cohn, "Comics, linguistics, and visual language: the past and the future of a field", in F. Bramlett (Ed.), Linguistics and the study of comics, New York: Palgrave MacMillan, 2012, pp. 92-118. DOI: 10.1057/9781137004109 5.

[19] H.Erhan, B. Yousuf, and B. Berry, 'Teaching Spatial Thinking in Design Computation Contexts: Challenges and Opportunities", in N. $\mathrm{Gu}$, \& X. Wang (Eds.) Computational Design Methods and Technologies: Applications in CAD, CAM and CAE Education, 2012, pp. 365-389 (chapter 21). Hershey, PA: Information Science Reference. DOI: 10.4018/978-1-61350-180-1.ch021.

[20] A. Doxiadis, C. H. Papadimitriou, Logicomix: An Epic Search for Truth, London: Bloomsbury Publishing PLC, 2009, 352 p. 